\title{
Coexistence of mitochondrial DNA and $\beta$ myosin heavy chain mutations in hypertrophic cardiomyopathy with late congestive heart failure
}

E Arbustini, R Fasani, P Morbini, M Diegoli, M Grasso, B Dal Bello, E Marangoni, P Banfi, N Banchieri, O Bellini, G Comi, J Narula, C Campana, A Gavazzi, C Danesino, $M$ Viganò

Department of
Cardiovascular
Pathology and
Molecular Diagnostics,
University School of
Medicine of
Pavia-Istituto di
Ricovero e Cura a
Carattere Scientifico
(IRCCS) Policlinico
San Matteo, Pavia,
Italy
E Arbustini
R Fasani
P Morbini
M Diegoli
M Grasso
B Dal Bello
N Banchieri
O Bellini

Department of Cardiology, University School of Medicine of Pavia

C Campana

A Gavazzi

Department of Cardiac Surgery, University School of Medicine of Pavia

$M$ Viganò

Department of Human Genetics, University School of Medicine of Pavia

C Danesino

Department of Cardiology and Neurology, Ospedale Maggiore, Lodi, Italy E Marangon

Centro Dino Ferrari, Istituto di Clinica Neurologica,

Università di Milano, IRCCS Ospedale Milano, Italy

P Banfi

Allegheny University of Health Sciences, Philadelphia, USA

J Narula

Correspondence to: Dr E Arbustini, Istituto di Anatomia Patologica, Via Forlanini 16, 27100 Pavia, Italy.

Accepted for publication 4 March 1998

\begin{abstract}
Objective-To investigate the possible coexistence of mitochondrial DNA (mtDNA) mutations in patients with $\beta$ myosin heavy chain ( $\beta M H C)$ linked hypertrophic cardiomyopathy (HCM) who develop congestive heart failure.

Design-Molecular analysis of $\beta M H C$ and mtDNA gene defects in patients with HCM. Setting-Cardiovascular molecular diagnostic and heart transplantation reference centre in north Italy.

Patients-Four patients with HCM who underwent heart transplantation for end stage heart failure, and after pedigree analysis of 60 relatives, eight additional affected patients and 27 unaffected relatives. A total of 111 unrelated healthy adult volunteers served as controls. Disease controls included an additional 27 patients with HCM and 102 with dilated cardiomyopathy.
\end{abstract}

Intervention-Molecular analysis of DNA from myocardial and skeletal muscle tissue and from peripheral blood specimens.

Main outcome measures-Screening for mutations in BMHC (exons 3-23) and mtDNA tRNA $(n=22)$ genes with denaturing gradient gel electrophoresis or single strand conformational polymorphism followed by automated DNA sequencing. Results-One proband (kindred A) (plus seven affected relatives) had arginine 249 glutamine (Arg249Gln) BMHC and heteroplasmic mtDNA tRNAIle A4300G mutations. Another unrelated patient (kindred B) with sporadic HCM had identical mutations. The remaining two patients (kindred C), a mother and son, had a novel BMHC mutation (lysine 450 glutamic acid) (Lys450Glu) and a heteroplasmic missense $($ T9957C, phenylalanine (Phe)->leucine (Leu)) mtDNA mutation in subunit III of the cytochrome $C$ oxidase gene. The amount of mutant mtDNA was higher in the myocardium than in skeletal muscle or peripheral blood and in affected patients than in asymptomatic relatives. Mutations were absent in the controls. Pathological and biochemical characteristics of patients with mutations Arg249Gin plus A4300G (kindreds $A$ and B) were identical, but different from those of the two patients with Lys450Glu plus T9957C(Phe->Leu) mutations (kindred
C). Cytochrome C oxidase activity and histoenzymatic staining were severely decreased in the two patients in kindreds $A$ and $B$, but were unaffected in the two in kindred $\mathrm{C}$.

Conclusions-BMHC gene and mtDNA mutations may coexist in patients with HCM and end stage congestive heart failure. Although BMHC gene mutations seem to be the true determinants of HCM, both mtDNA mutations in these patients have known prerequisites for pathogenicity. Coexistence of other genetic abnormalities in BMHC linked HCM, such as mtDNA mutations, may contribute to variable phenotypic expression and explain the heterogeneous behaviour of HCM.

(Heart 1998;80:548-558)

Keywords: $\beta$ myosin heavy chain; mitochondrial DNA; hypertrophic cardiomyopathy; oxidative phosphorylation; congestive heart failure

Familial hypertrophic cardiomyopathy (HCM) is a genetically transmitted heart disease with a dominant mode of inheritance. ${ }^{1}$ The disease is caused by more than 50 mutant alleles. Most mutations have been identified in the $\beta$ myosin heavy chain ( $\beta$ MHC) gene, ${ }^{2-11}$ and, less frequently, in the genes for cardiac myosin binding protein $\mathrm{C},{ }^{12} \alpha$ tropomyosin, ${ }^{13}{ }^{14}$ cardiac troponin $T,{ }^{1315}$ and myosin light chain. ${ }^{16}$ Although these mutations in genes encoding for sarcomeric proteins have been causally linked to HCM, it is not clear why the clinical spectrum of HCM is so heterogeneous, even within a single family.

Recent studies have reported that maternally inherited, non-X linked HCMs are associated with defects in mitochondrial oxidative metabolism, which are primarily due to mitochondrial DNA (mtDNA) deletions and to mtDNA transfer RNA (tRNA) point mutations. ${ }^{17-24}$ Defective mitochondrial function may be associated with a reduced energy supply to the heart and may contribute to congestive heart failure $(\mathrm{CHF})$, particularly in patients with a severely hypertrophic myocardium. This hypothesis is supported by the association of mtDNA mutation with dilated cardiomyopathy. ${ }^{25}$

In the present study, we describe the coexistence of pathological mutations in the $\beta M H C$ gene and mtDNA in three families of four patients with HCM who developed $\mathrm{CHF}$ requiring heart transplantation. DNA was obtained from heart, skeletal muscle, and 


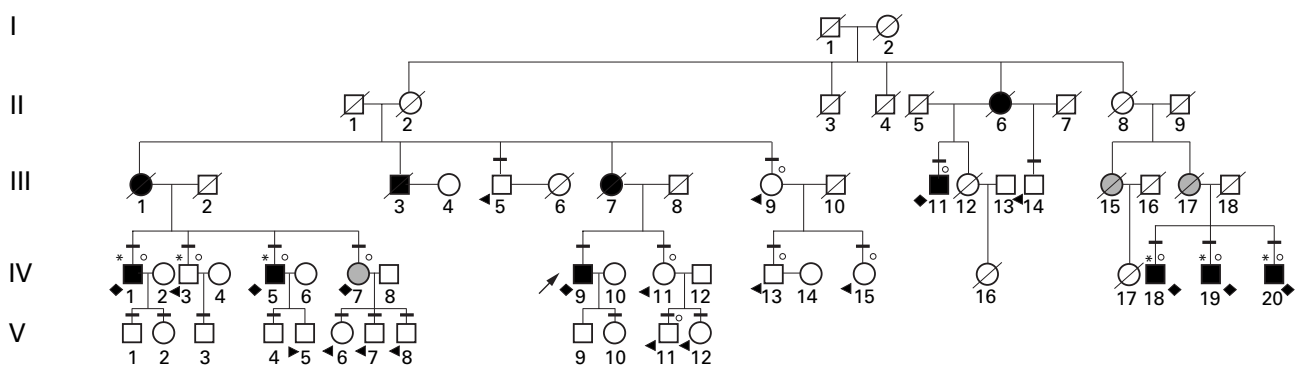

Family B

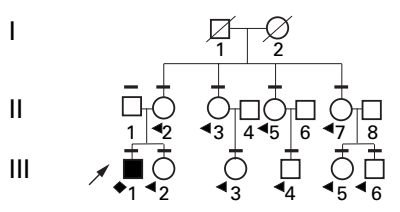

\begin{tabular}{|l|}
\hline Hypomagnesaemia \\
* Pes cavus \\
mtDNA mutation \\
- $\beta$-MHC gene mutation \\
Available for molecular \\
\\
analysis
\end{tabular}

Family C

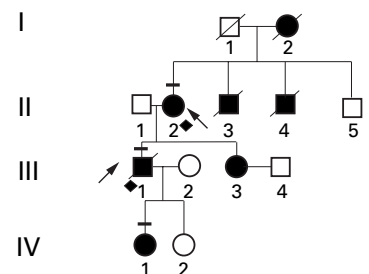

Figure 1 Three pedigrees showing familial ( $A$ and $C$ ) and non-familial $(B)$ hypertrophic cardiomyopathy (HCM) with late dilating congestive evolution. Solid symbols indicate affected individuals, both symptomatic and asymptomatic. Horizontal bars indicate individuals whose DNA was tested. Probands are indicated by arrows. Family A. I: subjects 1 and 2 died of non-cardiac causes in old age; II: subjects 1,2, 5, and 7-9 died of non-cardiac causes, subjects 3 and 4 may possibly have died of non-cardiac causes, and subject 6 died suddenly of heart disease at 48 years of age; III: subjects 1, 3, and 7 were clinically documented by medical records as affected by cardiomyopathy and died of congestive heart failure (III-1 and III-3) and of sudden death (III-7) at 47, 50, and 48 years of age, respectively; subjects 15 and 17 were probably affected by heart disease but were referred to as having died of non-cardiac causes; subjects 2, 6, 8, 10,12, 16, and 18 died of non-cardiac causes in old age; IV: subject 16 died of hydrocephalia/ventricular malformation at 7 years of age; subject 17 died of unspecified malformations at 12 years of age. (Table 1 summarises information about other family members.) Family B. I: subjects 1 and 2 died of non-cardiac causes in old age. (Table 1 summarises information about other family members.) Family C. I: subject 1 died of non-cardiac causes in old age; subject 2 died of heart disease with congestive heart failure at 59 years of age; II: subject 3 died of "cardiomyopathy" with congestive heart failure at 18 years of age; subject 4 died suddenly at 32 years of age; III: subject 1 died after heart transplantation at 31 years of age; subject 3 had HCM with supraventricular arrhythmias; IV: subject 1 (8 years old) is affected by HCM.

peripheral blood specimens of these four patients, and another affected relative, as well as from blood of 36 other relatives. The findings are compared with those of 111 healthy volunteers.

\section{Patients and methods}

Four patients from three families (A, B, and C) underwent orthotopic heart transplantation for $\mathrm{CHF}$ secondary to HCM. Pedigree and genetic analyses of the three kindreds were performed (fig 1 and table 1). Detailed historical and subsequent clinical investigation of 60 maternal relatives of the kindreds showed that 22 had HCM or were diagnosed as having HCM before death. Forty two maternal members are alive. Four relatives, in addition to the four probands, are symptomatic; four with echocardiographic asymmetrical septal hypertrophy on screening are asymptomatic and one relative has an isolated electrocardiographic abnormality. All living relatives were born to mothers with normal pregnancies and delivered to healthy, non-consanguineous parents.

\section{KINDREDS A-C, PATIENTS, AND MATERNAL}

RELATIVES

Family $A$

Patient A-IV-9, the proband, is a 44 year old man who underwent transplantation 11 years ago. The patient has been well except for recent chronic renal failure. Two cousins also have
HCM. The first cousin (A-IV-1) is a 46 year old man who presented with recurrent sustained ventricular tachyarrhythmias three years earlier: HCM was diagnosed. He was refractory to various antiarrhythmic drugs and an automatic cardioverter defibrillator was implanted. He also has pes cavus and persistent hypomagnesaemia with excessive renal magnesium loss. There is no evidence of CHF. The second cousin, A-IV-5, is a 42 year old man who was diagnosed as having HCM two years ago. He also has isolated persistent hypomagnesaemia with inappropriate renal magnesium loss and pes cavus. He is clinically stable and is receiving $\beta$ blockers.

Four additional asymptomatic individuals with asymmetrical HCM (A-III-11, and A-IV18-19-20) were identified by screening of the remaining family members. All four have hypomagnesaemia and three have pes cavus. Another relative (A-IV-7) has isolated T wave inversion in leads $\mathrm{V} 1-\mathrm{V} 3$ and hypomagnesaemia. Living, unaffected maternal relatives without cardiovascular problems were also evaluated: hypomagnesaemia was found in five and pes cavus in one (table 1). No history of cardiac or skeletal muscle disease was reported in paternal relatives.

\section{Family $B$}

A 31 year old man (B-III-1) underwent cardiac transplantation for end stage $\mathrm{CHF}$ 
Table 1 Clinical, biochemical, and molecular characteristics of the 40 examined subjects from the three families $A$, $B$, and $C$

\begin{tabular}{|c|c|c|c|c|c|c|c|c|c|c|c|c|c|c|}
\hline & \multirow[b]{2}{*}{ Sex/age } & \multirow[b]{2}{*}{$\begin{array}{l}\text { ECG } \\
\text { abnormalities }\end{array}$} & \multirow[b]{2}{*}{$\begin{array}{l}\text { Echo IVS } \\
\text { (cm) }\end{array}$} & \multirow{2}{*}{$\begin{array}{l}\text { Echo PW } \\
\text { (cm) }\end{array}$} & \multirow[b]{2}{*}{$\begin{array}{l}\text { Echo LVD } \\
\text { (cm) }\end{array}$} & \multirow{2}{*}{$\begin{array}{l}\text { Echo } \\
\text { LVEF (\%) }\end{array}$} & \multirow[b]{2}{*}{$\begin{array}{l}\text { Echo IVS } \\
\text { hypertrophy }\end{array}$} & \multirow{2}{*}{$\begin{array}{l}\text { Hypo } \\
M g\end{array}$} & \multirow[b]{2}{*}{$\begin{array}{l}\text { Pes } \\
\text { cavus }\end{array}$} & \multicolumn{3}{|c|}{$m t D N A(\%)$} & \multirow{2}{*}{$\begin{array}{l}\beta M H C \\
\text { mutation }\end{array}$} & \multirow{2}{*}{$\begin{array}{l}\text { Other } \\
\text { cardiovascular } \\
\text { or extracardiac } \\
\text { abnormalities }\end{array}$} \\
\hline & & & & & & & & & & Blood & Heart & Muscle & & \\
\hline \multicolumn{15}{|c|}{ Family A } \\
\hline III-5 & $\mathrm{M} / 70$ & RBBB-LVH & 1.3 & 1.3 & 3.8 & 65 & Diffuse & - & - & 65 & & & - & $\begin{array}{l}\text { Hypertension, } \\
\text { COPD }\end{array}$ \\
\hline III-9 & $\mathrm{F} / 55$ & - & 1.1 & 1.1 & 5.2 & 60 & / & + & - & 70 & & & - & - \\
\hline III-11 & $\mathrm{M} / 67$ & LVH & 1.4 & 1.1 & 4.7 & 75 & Mid-septum & + & $\neq$ & 88 & & & + & Stroke \\
\hline III- 14 & $\mathrm{M} / 62$ & - & 1.1 & 1.0 & 4.4 & 65 & / & - & - & 58 & & & - & - \\
\hline IV-1 & $\mathrm{M} / 46$ & LVH & 1.5 & 0.9 & 7.3 & 40 & $\begin{array}{l}\text { Mid-apical } \\
\text { septum }\end{array}$ & + & + & 90 & 95 & 82 & + & - \\
\hline IV-3 & $\mathrm{M} / 45$ & - & 1.1 & 1.0 & 5.3 & 65 & / & + & + & 70 & & & - & - \\
\hline IV-5 & $\mathrm{M} / 42$ & $\mathrm{LVH}$ & 1.6 & 1.3 & 4.9 & 45 & $\begin{array}{l}\text { Mid-apical } \\
\text { septum }\end{array}$ & + & + & 92 & & & + & - \\
\hline IV-7 & $\mathrm{F} / 39$ & $\begin{array}{l}\text {-ve T waves } \\
\mathrm{V} 1-\mathrm{V} 3\end{array}$ & 0.9 & 0.8 & 4.9 & 55 & / & + & - & 70 & & & + & $\begin{array}{l}\text { Kidney } \\
\text { malformation* } \\
\text { Transient } \\
\text { amaurosis }\end{array}$ \\
\hline IV-9 & $\mathrm{M} / 44$ & LVH & 1.8 & 1.7 & 8.1 & 22 & Diffuse & $\neq \neq$ & - & 95 & 98 & 85 & + & $\begin{array}{l}\text { Late renal } \\
\text { failure post-HT }\end{array}$ \\
\hline IV-11 & $\mathrm{F} / 36$ & IRBBB & 0.9 & 0.8 & 4.2 & 60 & l & + & - & 65 & & & - & - \\
\hline IV-13 & $\mathrm{M} / 36$ & - & 1.1 & 1.0 & 5.2 & 65 & l & + & - & 50 & & & - & - \\
\hline IV-15 & $\mathrm{F} / 31$ & - & 0.9 & 0.8 & 4.1 & 60 & l & - & - & 55 & & & - & - \\
\hline IV-18 & $\mathrm{M} / 58$ & LVH & 1.3 & 1.1 & 4.9 & 65 & Basal septum & + & + & 62 & & & + & $\begin{array}{l}\text { Prior non-Q } \\
\text { wave MI }\end{array}$ \\
\hline IV-19 & $\mathrm{M} / 56$ & - & 1.3 & 1.1 & 4.3 & 65 & $\begin{array}{l}\text { Mid-basal } \\
\text { septum }\end{array}$ & + & + & 65 & & & + & Gout \\
\hline IV-20 & $\mathrm{M} / 50$ & $\mathrm{LVH}$ & 1.3 & 1.0 & 4.0 & 60 & Mid-septum & + & + & 60 & & & + & $\begin{array}{l}\text { Mild } \\
\text { hypertension }\end{array}$ \\
\hline V-1 & $\mathrm{M} / 18$ & IRBBB & 1.1 & 1.0 & 4.4 & 60 & I & - & - & I & & & - & - \\
\hline $\mathrm{V}-2$ & $\mathrm{~F} / 14$ & - & 0.9 & 0.9 & 4.4 & 60 & l & - & - & l & & & - & - \\
\hline $\mathrm{V}-3$ & $M / 15$ & - & 0.8 & 0.8 & 4.8 & 60 & I & - & - & I & & & - & - \\
\hline $\mathrm{V}-4$ & $\mathrm{M} / 15$ & - & 0.8 & 0.8 & 4.5 & 55 & I & - & - & l & & & - & - \\
\hline$V-5$ & $\mathrm{M} / 13$ & - & 0.8 & 0.8 & 4.5 & 60 & / & - & - & / & & & + & $\begin{array}{l}\text { Bilateral pes } \\
\text { contortus }\end{array}$ \\
\hline V-6 & $\mathrm{F} / 25$ & - & 0.9 & 0.7 & 5.0 & 65 & / & + & - & 78 & & & - & - \\
\hline $\mathrm{V}-7$ & $\mathrm{M} / 17$ & - & 1.1 & 1.1 & 4.5 & 60 & / & - & - & 70 & & & + & - \\
\hline $\mathrm{V}-8$ & $\mathrm{M} / 15$ & - & 1.0 & 1.0 & 4.6 & 65 & / & - & - & 75 & & & - & - \\
\hline$V-10$ & $\mathrm{~F} / 12$ & - & 0.9 & 0.8 & 4.0 & 60 & / & - & - & I & & & - & - \\
\hline$V-11$ & $\mathrm{M} / 18$ & - & 1.1 & 1.1 & 5.3 & 60 & I & + & - & 65 & & & - & Left renal cyst \\
\hline $\mathrm{V}-12$ & $\mathrm{~F} / 10$ & - & 0.9 & 0.8 & 4.0 & 65 & l & + & - & 70 & & & - & - \\
\hline \multicolumn{15}{|c|}{ Family B } \\
\hline II-1 & $\mathrm{M} / 57$ & - & 0.7 & 0.9 & 5.3 & 63 & l & - & - & - & & & - & - \\
\hline II-2 & $\mathrm{F} / 54$ & - & 0.9 & 0.9 & 4.4 & 60 & I & - & - & 75 & & & - & - \\
\hline II-3 & $\mathrm{F} / 51$ & - & 0.9 & 0.1 & 5.0 & 56 & l & - & - & 70 & & & - & $\begin{array}{l}\text { Mild mitral } \\
\text { valve prolapse }\end{array}$ \\
\hline II-5 & $\mathrm{F} / 58$ & - & 0.75 & 0.75 & 4.0 & 52 & I & - & - & 68 & & & - & - \\
\hline II-7 & $\mathrm{F} / 42$ & - & 0.75 & 0.85 & 4.5 & 55 & I & - & - & 73 & & & - & - \\
\hline III-1 & M31 & LVH & 1.3 & 1.4 & 7.8 & 20 & Diffuse & - & - & 76 & 95 & 85 & + & - \\
\hline III-2 & $\mathrm{F} / 21$ & - & 0.9 & 0.8 & 4.8 & 48 & I & - & - & 82 & & & - & - \\
\hline III-3 & F30 & - & 1.05 & 1.05 & 4.5 & 61 & l & - & - & 72 & & & - & - \\
\hline III-4 & $\mathrm{M} / 31$ & - & 0.8 & 1 & 5.1 & 60 & l & - & - & 80 & & & - & - \\
\hline III-5 & $\mathrm{F} / 16$ & - & 0.9 & 0.75 & 4.3 & 58 & l & - & - & 78 & & & - & - \\
\hline III-6 & $\mathrm{M} / 48$ & - & 1.15 & 1.1 & 4.8 & 70 & I & - & - & 70 & & & - & - \\
\hline \multicolumn{15}{|c|}{ Family C } \\
\hline II-2 & $\mathrm{F} / 55$ & AVB III/PM & 1.0 & 0.7 & 3.8 & 20 & Diffuse septal & - & - & 45 & 64 & 70 & + & $\begin{array}{l}\text { Height } 145 \mathrm{~cm} \text {, } \\
\text { weight } 40 \mathrm{~kg}\end{array}$ \\
\hline III-1 & $M / 31$ & AVB III/PM & 1.0 & 0.8 & 6.0 & 15 & Mid-septum & - & - & 48 & 92 & 82 & + & $\begin{array}{l}\text { Height } 174 \mathrm{~cm} \text {, } \\
\text { weight } 63 \mathrm{~kg}\end{array}$ \\
\hline IV-1 & $\mathrm{F} / 9$ & LVH & 2.2 & 0.7 & 3.1 & & Septal diffuse & - & - & - & - & - & + & $\begin{array}{l}\text { Height } 135 \mathrm{~cm} \text {, } \\
\text { weight } 38 \mathrm{~kg}\end{array}$ \\
\hline
\end{tabular}

$\neq$ Not evaluated because of previous stroke; $\neq \neq$ Not evaluated because of renal failure; ${ }^{\star}$ Right renal hypoplasia, double pelvis.

IVS, interventricular septum; PW, posterior wall; LVD, left ventricular diameter; LVEF, left ventricular ejection fraction; Hypo Mg, hypomagnesaemia; RBBB, right bundle branch block; LVH, left ventricular hypertrophy ( $\mathrm{S}$ in V1 and in V5 > $35 \mathrm{~mm}$ ); IRBBB, incomplete right bundle branch block; COPD, chronic obstructive pulmonary disease; HT, heart transplantation; M, myocardial infarction; AVB atrioventricular block; PM, pacemaker.

secondary to HCM 12 years after HCM was diagnosed. Serum magnesium levels were within normal range. He is doing well five years after transplantation. There is no clinical or laboratory evidence of HCM, hypomagnesaemia, or pes cavus in either maternal or paternal relatives.

Family $C$

Two patients, a mother and son, underwent heart transplantation for end stage heart failure complicating HCM. HCM was diagnosed in the mother (C-II-2, 55 years old) 19 years earlier. A permanent pacemaker was implanted for complete atrioventricular block seven years earlier. She is doing well after heart transplan- tation. Her son (C-III-1) at the age of 17 also underwent pacemaker implantation due to complete atrioventricular block. He underwent cardiac transplantation when aged 31 years but died of sepsis three days after transplantation. One of his daughters and one sister also have HCM. The affected daughter (C-IV-1, 9 years old) has supraventricular arrhythmias and is receiving calcium channel blockers. His sister (C-III-3, 31 years old) also has asymmetrical HCM; she recently developed supraventricular tachycardia with a concealed atrioventricular accessory pathway and underwent transcatheter radiofrequency ablation. She is doing well and is receiving calcium channel blockers. 
Table 2 Primer sequence and polymerase chain reaction (PCR) conditions

\begin{tabular}{|c|c|c|c|c|}
\hline $\begin{array}{l}\beta \text { myosin } \\
\text { gene exon }\end{array}$ & $\begin{array}{l}\text { Position } \\
\text { map }\end{array}$ & $\begin{array}{l}P C R \\
\text { fragment }\end{array}$ & Primers & PCR conditions \\
\hline 3 & $5656-5988$ & $332 \mathrm{bp}$ & $\begin{array}{l}\text { F 5' AGGGAGGGAAGGGAAGATG 3'; } \\
\text { R 5' TACCCCTCTCTGTCCACCCA 3' }\end{array}$ & $94^{\circ} \mathrm{C} 30^{\prime \prime}, 63^{\circ} \mathrm{C} 20^{\prime \prime},, 72^{\circ} \mathrm{C} 20^{\prime \prime}\left(30\right.$ cycles); $72^{\circ} \mathrm{C} 6^{\prime}$ \\
\hline 4 & $6145-6428$ & $283 \mathrm{bp}$ & $\begin{array}{l}\text { F 5' TCACTCACCAACTCCTAACC 3'; } \\
\text { R'5'GGGTGGACATGGATGGAGCA 3' }\end{array}$ & $94^{\circ} \mathrm{C} 20^{\prime \prime}, 63^{\circ} \mathrm{C} 15^{\prime \prime}, 72^{\circ} \mathrm{C} 20^{\prime \prime}$ (30 cycles); $72^{\circ} \mathrm{C} 6^{\prime}$ \\
\hline 5 & $6588-6895$ & $307 \mathrm{bp}$ & $\begin{array}{l}\text { F 5' TCTAACTTCCAAAATCACCA 3'; } \\
\text { R 5' TCTCCCTTCCTTCTCCCTCT 3' }\end{array}$ & $94^{\circ} \mathrm{C} 20^{\prime \prime}, 54^{\circ} \mathrm{C} 30^{\prime \prime}, 72^{\circ} \mathrm{C} 20^{\prime \prime}(30$ cycles $) ; 72^{\circ} \mathrm{C} 6^{\prime}$ \\
\hline 6 & $6882-7028$ & $146 \mathrm{bp}$ & $\begin{array}{l}\text { F 5' GAGAAGCCCCACGAGAGCAT 3'; } \\
\text { R 5' TGGAGGCTGGGATCAGGGAG 3' }\end{array}$ & $94^{\circ} \mathrm{C} 20^{\prime \prime}, 63^{\circ} \mathrm{C} 15^{\prime \prime}, 72^{\circ} \mathrm{C} 20^{\prime \prime}\left(30\right.$ cycles); $72^{\circ} \mathrm{C} 6^{\prime}$ \\
\hline 7 & $7516-7760$ & $244 \mathrm{bp}$ & $\begin{array}{l}\text { F 5' CTGATTTGAGGCTTCCTGGT 3'; } \\
\text { R 5' AAGAAGGAGGCAGGTGAGAG 3' }\end{array}$ & $94^{\circ} \mathrm{C} 20^{\prime \prime}, 63^{\circ} \mathrm{C} 15^{\prime \prime}, 72^{\circ} \mathrm{C} 20^{\prime \prime}(30$ cycles $) ; 72^{\circ} \mathrm{C} 6^{\prime}$ \\
\hline 8 & $7720-7960$ & $240 \mathrm{bp}$ & $\begin{array}{l}\text { F 5' CGCAGAAGGGAGGGAGAAGA 3'; } \\
\text { R 5' AGTGGTGATGAGTTGGGGGA 3' }\end{array}$ & $94^{\circ} \mathrm{C} 30^{\prime \prime}, 63^{\circ} \mathrm{C} 20^{\prime \prime}, 72^{\circ} \mathrm{C} 20^{\prime \prime}(30$ cycles $) ; 72^{\circ} \mathrm{C} 6^{\prime}$ \\
\hline 9 & $7891-8114$ & $223 \mathrm{bp}$ & $\begin{array}{l}\text { F 5' GGACTIGGACTGGTGGAGGA 3'; } \\
\text { R 5' GGGGAGAGAGAGAGAGGTCA 3' }\end{array}$ & $94^{\circ} \mathrm{C} 10^{\prime \prime}, 94^{\circ} \mathrm{C} 30^{\prime \prime}, 63^{\circ} \mathrm{C} 20^{\prime \prime}, 72^{\circ} \mathrm{C} 20^{\prime \prime}\left(30\right.$ cycles); $72^{\circ} \mathrm{C} 6^{\prime}$ \\
\hline 10 & $8384-8609$ & $225 \mathrm{bp}$ & $\begin{array}{l}\text { F 5' ATTAATTTTCCTTGTGCCCA 3'; } \\
\text { R 5' CAAGCAGAGGGGACCAGGTT 3' }\end{array}$ & $94^{\circ} \mathrm{C} 20^{\prime \prime},, 56^{\circ} \mathrm{C} 30^{\prime \prime},, 72^{\circ} \mathrm{C} 20^{\prime \prime}(30$ cycles $) ; 72^{\circ} \mathrm{C} 6^{\prime}$ \\
\hline 11 & $8724-8962$ & $238 \mathrm{bp}$ & $\begin{array}{l}\text { F 5' CCTCCATGAGAGCCGGGGGC 3'; } \\
\text { R 5' CTGCTTTTGGACCCCTGTTT 3' }\end{array}$ & $94^{\circ} \mathrm{C} 20^{\prime \prime},, 63^{\circ} \mathrm{C} 15^{\prime \prime},, 72^{\circ} \mathrm{C} 20^{\prime \prime}(30$ cycles $) ; 72^{\circ} \mathrm{C} 6^{\prime}$ \\
\hline 12 & $9479-9750$ & $271 \mathrm{bp}$ & $\begin{array}{l}\text { F 5' CTCACTTACCCATCATACTT 3'; } \\
\text { R 5' AGAAAAGAGAAGAGAGATAC 3' }\end{array}$ & $94^{\circ} \mathrm{C} 20^{\prime \prime},, 63^{\circ} \mathrm{C} 20^{\prime \prime},, 72^{\circ} \mathrm{C} 20^{\prime \prime}$ (30 cycles); $72^{\circ} \mathrm{C} 6^{\prime}$ \\
\hline 13 & $10023-10308$ & $285 \mathrm{bp}$ & $\begin{array}{l}\text { F 5'ACAGGCATGAACCACCACAC 3'; } \\
\text { R 5' GCAGGGTTGTTGGGAAGAGT 3' }\end{array}$ & $94^{\circ} \mathrm{C} 10^{\prime \prime}, 94^{\circ} \mathrm{C} 15^{\prime \prime}, 65^{\circ} \mathrm{C} 15^{\prime \prime}, 72^{\circ} \mathrm{C} 10^{\prime \prime}\left(30\right.$ cycles); $72^{\circ} \mathrm{C} 6^{\prime}$ \\
\hline 14 & $10266-10589$ & $323 \mathrm{bp}$ & $\begin{array}{l}\text { F 5' GTGGGATGAGAGTTTTCAAG 3'; } \\
\text { R 5' CTGCCTTCCCATGTCTGGTC 3' }\end{array}$ & $94^{\circ} \mathrm{C} 20^{\prime \prime}, 63^{\circ} \mathrm{C} 20^{\prime \prime}, 72^{\circ} \mathrm{C} 20^{\prime \prime}(30$ cycles $) ; 72^{\circ} \mathrm{C} 6^{\prime}$ \\
\hline 15 & $10693-11014$ & $321 \mathrm{bp}$ & $\begin{array}{l}\text { F 5' AGGCACAGTGGACGGGCACA 3'; } \\
\text { R 5' GGCTGCTATTTTGTCTATGG 3' }\end{array}$ & $94^{\circ} \mathrm{C} 30^{\prime \prime}, 63^{\circ} \mathrm{C} 20^{\prime \prime}, 72^{\circ} \mathrm{C} 20^{\prime \prime}\left(30\right.$ cycles); $72^{\circ} \mathrm{C} 6^{\prime}$ \\
\hline 16 & $11476-11914$ & $438 \mathrm{bp}$ & $\begin{array}{l}\text { F 5' TTGACCATAGAGCAGAATCC 3'; } \\
\text { R 5' CTCAGAACCTTGGGAGAATC 3' }\end{array}$ & $94^{\circ} \mathrm{C} 30^{\prime \prime}, 63^{\circ} \mathrm{C} 15^{\prime \prime}, 72^{\circ} \mathrm{C} 20^{\prime \prime}\left(30\right.$ cycles); $72^{\circ} \mathrm{C} 6^{\prime}$ \\
\hline 17 & $12068-12255$ & $187 \mathrm{bp}$ & $\begin{array}{l}\text { F 5'TCTTACTCACACCCTACCTC 3'; } \\
\text { R 5' GGGGCTGGGTGGGGTTGGGC 3' }\end{array}$ & $94^{\circ} \mathrm{C} 30^{\prime \prime}, 63^{\circ} \mathrm{C} 15^{\prime \prime}, 72^{\circ} \mathrm{C} 20^{\prime \prime}\left(30\right.$ cycles); $72^{\circ} \mathrm{C} 6^{\prime}$ \\
\hline 18 & $12498-12795$ & 297 bp & $\begin{array}{l}\text { F 5' CTTCTCCTCTCTTCTTCCTG 3'; } \\
\text { R 5' TGGTGGTAGGTAGGGAGAGT 3' }\end{array}$ & $94^{\circ} \mathrm{C} 20^{\prime \prime}, 61^{\circ} \mathrm{C} 20^{\prime \prime}, 72^{\circ} \mathrm{C} 20^{\prime \prime}\left(30\right.$ cycles); $72^{\circ} \mathrm{C} 6^{\prime}$ \\
\hline 19 & $13269-13540$ & $271 \mathrm{bp}$ & $\begin{array}{l}\text { F 5'AATCAGTGACAAAGCCAGGA 3'; } \\
\text { R 5' GCAGATCCCATTCCCATCAG 3' }\end{array}$ & $94^{\circ} \mathrm{C} 20^{\prime \prime}, 61^{\circ} \mathrm{C} 20^{\prime \prime}, 72^{\circ} \mathrm{C} 20^{\prime \prime}$ (30 cycles); $72^{\circ} \mathrm{C} 6^{\prime}$ \\
\hline 20 & $13521-13819$ & 298 bp & $\begin{array}{l}\text { F 5' CCTGATGGGAATGGGATCTG 3'; } \\
\text { R 5' ATGATTACAACAGGAAAAGC 3' }\end{array}$ & $94^{\circ} \mathrm{C} 20^{\prime \prime}, 61^{\circ} \mathrm{C} 20^{\prime \prime}, 72^{\circ} \mathrm{C} 20^{\prime \prime}\left(30\right.$ cycles); $72^{\circ} \mathrm{C} 6 !$ \\
\hline 21 & $13941-14226$ & $285 \mathrm{bp}$ & $\begin{array}{l}\text { F 5' TCCTAAGGTAATCCCCACCA 3'; } \\
\text { R 5' CTTTTTTTCCTGACACTGCC 3' }\end{array}$ & $94^{\circ} \mathrm{C} 20^{\prime \prime}, 63^{\circ} \mathrm{C} 15^{\prime \prime}, 72^{\circ} \mathrm{C} 20^{\prime \prime}(30$ cycles $) ; 72^{\circ} \mathrm{C} 6$ \\
\hline 22 & $14358-14720$ & $362 \mathrm{bp}$ & $\begin{array}{l}\text { F 5' AGGTAGGAAGGAGGCAGAGG 3'; } \\
\text { R 5' GGGTCAAGGTCAGTATGGTC 3' }\end{array}$ & $94^{\circ} \mathrm{C} 20^{\prime \prime}, 63^{\circ} \mathrm{C} 15^{\prime \prime}, 72^{\circ} \mathrm{C} 20^{\prime \prime}\left(30\right.$ cycles); $72^{\circ} \mathrm{C} 6^{\prime}$ \\
\hline 23 & $15202-15594$ & 392 bp & $\begin{array}{l}\text { F 5' TGATGTGCCTCTCCTTCCCT 3'; } \\
\text { R 5' GGGTCAAGGTCAGTATGGTC 3' }\end{array}$ & $94^{\circ} \mathrm{C} 30^{\prime \prime}, 63^{\circ} \mathrm{C} 20^{\prime \prime}, 72^{\circ} \mathrm{C} 20^{\prime \prime}$ (30 cycles); $72^{\circ} \mathrm{C} 6^{\prime}$ \\
\hline \multicolumn{5}{|l|}{$\begin{array}{l}\text { Mitochondrial } \\
\text { tRNA }\end{array}$} \\
\hline Phe & $503-717$ & $214 \mathrm{bp}$ & $\begin{array}{l}\text { F 5' ATCCTACCCAGCACACACAC 3'; } \\
\text { R 5' ACTGGAACGGGGATGCTTGC 3' }\end{array}$ & $94^{\circ} \mathrm{C} 30^{\prime \prime}, 63^{\circ} \mathrm{C} 20^{\prime \prime}, 72^{\circ} \mathrm{C} 30^{\prime \prime}(25$ cycles $) ; 72^{\circ} \mathrm{C} 6^{\prime}$ \\
\hline Val & $1521-1751$ & $230 \mathrm{bp}$ & $\begin{array}{l}\text { F 5' TTAACTAAAACCCCTACGCA 3'; } \\
\text { R 5' CGCCTATACTTTATTTGGGT 3' }\end{array}$ & $94^{\circ} \mathrm{C} 30^{\prime \prime}, 58^{\circ} \mathrm{C} 20^{\prime \prime}, 72^{\circ} \mathrm{C} 30^{\prime \prime}$ (25 cycles); $72^{\circ} \mathrm{C} 6^{\prime}$ \\
\hline $\mathrm{Leu}^{\text {uur }}$ & $3168-3389$ & $221 \mathrm{bp}$ & $\begin{array}{l}\text { F 5' CCCCCGTAAATGATATCATC 3'; } \\
\text { R 5' GAATTTTTCGTTCGGTAAGC 3' }\end{array}$ & $94^{\circ} \mathrm{C} 30^{\prime \prime}, 58^{\circ} \mathrm{C} 20^{\prime \prime}, 72^{\circ} \mathrm{C} 30^{\prime \prime}(25$ cycles $) ; 72^{\circ} \mathrm{C} 6^{\prime}$ \\
\hline Ile, Gln ${ }^{c}, \mathrm{Met}^{\mathrm{f}}$ & $4180-4533$ & $353 \mathrm{bp}$ & $\begin{array}{l}\text { F 5' AACTTCCTACCACTCACCCT 3'; } \\
\text { R 5' CGCTGTGATGAGTGTGCCTG 3' }\end{array}$ & $94^{\circ} \mathrm{C} 30^{\prime \prime}, 62^{\circ} \mathrm{C} 20^{\prime \prime}, 72^{\circ} \mathrm{C} 30^{\prime \prime}(25$ cycles $) ; 72^{\circ} \mathrm{C} 6^{\prime}$ \\
\hline $\begin{array}{l}\text { Trp, Alac } \\
\text { Asn }^{\mathrm{c}}, \mathrm{Cys}^{\mathrm{c}}, \mathrm{Tyr}^{\mathrm{c}}\end{array}$ & $5450-5958$ & 502 bp & $\begin{array}{l}\text { F 5'CACACTCATCGCCCTTACCA 3'; } \\
\text { R 5' TAGTGTTCCAATGTCTTTGT 3' }\end{array}$ & $94^{\circ} \mathrm{C} 30^{\prime \prime}, 58^{\circ} \mathrm{C} 20^{\prime \prime}, 72^{\circ} \mathrm{C} 30^{\prime \prime}$ (25 cycles); $72^{\circ} \mathrm{C} 6^{\prime}$ \\
\hline Ser ${ }^{\text {ucn }}$, Asp & $7375-7633$ & $258 \mathrm{bp}$ & $\begin{array}{l}\text { F 5' ACCTGGAGTGACTATATGGA 3'; } \\
\text { R 5' ATAGGGGAAGTAGCGTCTTG 3' }\end{array}$ & $94^{\circ} \mathrm{C} 30^{\prime \prime}, 62^{\circ} \mathrm{C} 20^{\prime \prime}, 72^{\circ} \mathrm{C} 30^{\prime \prime}(25$ cycles $) ; 72^{\circ} \mathrm{C} 6^{\prime}$ \\
\hline Lys & $8199-8447$ & 248 bp & $\begin{array}{l}\text { F 5' GTTTCATGCCCATCGTCCTA 3'; } \\
\text { R 5' TTTTAGTTGGGTGATGAGGA 3' }\end{array}$ & $94^{\circ} \mathrm{C} 30^{\prime \prime}, 58^{\circ} \mathrm{C} 20^{\prime \prime}, 72^{\circ} \mathrm{C} 30^{\prime \prime}(25$ cycles $) ; 72^{\circ} \mathrm{C} 6^{\prime}$ \\
\hline Gly & $9923-10150$ & $227 \mathrm{bp}$ & $\begin{array}{l}\text { F 5' CTGATACTGGCATTTTGTAG 3'; } \\
\text { R 5' TGTAGCCGTTGAGTTGTGGT 3' }\end{array}$ & $94^{\circ} \mathrm{C} 30^{\prime \prime}, 58^{\circ} \mathrm{C} 20^{\prime \prime}, 72^{\circ} \mathrm{C} 30^{\prime \prime}$ ( 25 cycles); $72^{\circ} \mathrm{C} 6^{\prime}$ \\
\hline Arg & $10320-10555$ & $235 \mathrm{bp}$ & $\begin{array}{l}\text { F 5' GTTATGTCATCCCTCTTATT 3'; } \\
\text { R 5' AGGATATGAGGTGTGAGCGA 3' }\end{array}$ & $94^{\circ} \mathrm{C} 30^{\prime \prime}, 58^{\circ} \mathrm{C} 20^{\prime \prime}, 72^{\circ} \mathrm{C} 30^{\prime \prime}(25$ cycles $) ; 72^{\circ} \mathrm{C} 6^{\prime}$ \\
\hline His, Ser ${ }^{\text {agy }}$, Leu ${ }^{\text {cun }}$ & $12042-12403$ & $361 \mathrm{bp}$ & $\begin{array}{l}\text { F 5' CCTCATTCACACGAGAAAAC 3'; } \\
\text { R 5' GGTGGTAAGGATGGGGGGAA 3' }\end{array}$ & $94^{\circ} \mathrm{C} 30^{\prime \prime}, 58^{\circ} \mathrm{C} 20^{\prime \prime}, 72^{\circ} \mathrm{C} 30^{\prime \prime}$ (25 cycles); $72^{\circ} \mathrm{C} 6^{\prime}$ \\
\hline $\mathrm{Glu}^{\mathrm{c}}$ & $14565-14830$ & $265 \mathrm{bp}$ & $\begin{array}{l}\text { F 5' CACCGCTAACAATCAATACT 3'; } \\
\text { R 5' GAGTAGTTGGATGGGGTGGG 3' }\end{array}$ & $94^{\circ} \mathrm{C} 30^{\prime \prime}, 58^{\circ} \mathrm{C} 20^{\prime \prime}, 72^{\circ} \mathrm{C} 30^{\prime \prime}$ (25 cycles); $72^{\circ} \mathrm{C} 6^{\prime}$ \\
\hline Thr, Pro & $15810-16115$ & 305 bp & $\begin{array}{l}\text { F 5' CCGTACTATACTTCACAACA 3'; } \\
\text { R 5' GTGGCTGGCAGTAATGTACG 3' }\end{array}$ & $94^{\circ} \mathrm{C} 30^{\prime \prime}, 62^{\circ} \mathrm{C} 20^{\prime \prime}, 72^{\circ} \mathrm{C} 30^{\prime \prime}(25$ cycles $) ; 72^{\circ} \mathrm{C} 6^{\prime}$ \\
\hline
\end{tabular}

GENETIC ANALYSIS IN THE KINDREDS AND CONTROLS

Peripheral blood specimens, pectoral skeletal muscle, and explanted hearts from the four probands were used for total DNA extraction. Endomyocardial and skeletal muscle biopsy specimens of a cousin of proband A-IV-9 were also available. In addition, peripheral blood specimens from the seven patients with HCM in family A (including one isolated electrocardiographic abnormality) were obtained for molecular analysis. There were no additional patients with HCM in family B. Maternal con- sent for genetic analysis of patient C-IV-1 was obtained; other relatives declined genetic analysis. Peripheral blood specimens were also obtained from 18 of 19 unaffected maternal relatives of family $A$ and from the nine unaffected relatives of family $\mathrm{B}$. The only living, unaffected maternal relative in kindred C (C-III-5) could not be traced.

CONTROLS

In addition to 27 unaffected maternal relatives, total DNA was obtained from 111 unrelated healthy volunteers. They underwent clinical 
evaluation, electrocardiographic examination, and routine biochemical tests. Furthermore, as proband B was a sporadic occurrence, molecular analysis for paternity was also undertaken after voluntary consent was obtained from the family. The present four patients with HCM are from a total of 133 patients with cardiomyopathy, 31 with HCM, in which both mtDNA and $3 M H C$ gene exons 3-23 were analysed, and 102 with dilated cardiomyopathy with mitochondrial abnormalities, in which mtDNA was analysed.

MOLECULAR STUDIES OF $\beta$ MHC GENE AND MtDNA MUTATIONS

Total DNA was isolated from explanted tissue, skeletal muscle, and peripheral blood specimens of the four cardiac allograft recipients and of patient A-IV-1 with standardised protocols, ${ }^{26}$ as well as from peripheral blood specimens of the remaining asymptomatic, symptomatic, and unaffected maternal relatives and of 111 healthy controls. Specific oligonucleotide primers were designed and synthesised for analysis of exons $3-23$ of the $\beta M H C$ gene and for the $22 \mathrm{mtDNA}$ tRNA genes. The selected genomic regions were amplified by polymerase chain reaction (PCR) and subjected to denaturing gradient gel electrophoresis (DGGE) ${ }^{27}$ or single strand conformational polymorphism $(\mathrm{SSCP})^{28}$ for mutation screening. Abnormal DGGE or SSCP conformers underwent direct automated sequencing for specific identification of mutation sites. Restriction enzyme digestion was performed to assess mtDNA mutation heteroplasmia and to confirm heterozygous nuclear DNA mutations. Mismatch primers were generated when mutations did not gain or lose restriction enzyme sites. Enzyme digestion analysis was extended to all family relatives and the controls. Densitometric analysis was performed to estimate the amount of mutant mtDNA.

Synthesis of oligonucleotide primers

Primers were designed on the $\beta M H C$ gene $^{29}$ and $\mathrm{mtDNA}^{30}$ sequences (table 2). A 392 DNA-RNA synthesiser Perkin Elmer-ABI, Foster City, California, USA) was used to synthesise primers. They were purified with oligonucleotide purification cartridges (Perkin Elmer-ABI). Forward GC clamp primers were synthesised for DGGE.

Polymerase chain reaction

PCR products for $22 \mathrm{mt}$ tRNAs and exons $3-23$ of the $\beta M H C$ gene were generated from $0.1 \mu \mathrm{g}$ of total DNA in an automated thermal cycler (Cetus Gene Amp PRC System 9600; Perkin Elmer-ABI). Amplified fragments were separated by electrophoresis on $2 \%$ agarose gel and stained with ethidium bromide.

\section{$D G G E$ and SSCP}

Screening to detect base changes was performed with DGGE or SSCP as described previously. ${ }^{27}{ }^{28}$ Abnormal conformers were subjected to further analysis.

Automated DNA sequencing

DNA was purified with Centri-sep spin columns (Perkin Elmer-ABI) and used for cyclical sequencing with the Taq DyeDeoxy Terminator Cycle Sequencing Kit. Sequence gels were run in a 373A DNA Sequencer (Perkin Elmer-ABI) according to the manufacturer's recommendations.

Sequence comparisons and analyses were performed with Sequencer TM 3.0 software (Gene Codes Corporation, Ann Arbor, Michigan, USA).

\section{Measurement of mutant mtDNAs}

The two mtDNA mutants and one $\beta M H C$ gene mutant did not modify the restriction enzyme map. Therefore, we designed and synthesised mismatch primers that, in combination with each given mutation, gained or lost a restriction enzyme site. Heteroplasmy was assessed with restriction enzyme digestion on PCR products obtained from combination of mismatch primers (see Results) and corresponding reverse primers. Appropriate plasmid controls assessed complete restriction enzyme activity. Relative amounts of mutant mitochondrial genomes were measured with a BioProfile Bio-1D Blot Analyzer (Vilber Lourmat Biotechnology Division, Marne La Vallee, France).

HISTOPATHOLOGICAL, ULTRASTRUCTURAL, HISTOCHEMICAL, AND BIOCHEMICAL STUDIES

Hearts explanted at transplantation were macroscopically examined and photographed. The septal and left ventricular free walls were sampled for microscopic study.

\section{Histopathological studies}

Myocardial and skeletal muscle biopsy samples were fixed by immersion in buffered $10 \%$ formalin, dehydrated rapidly, and embedded in paraffin, cut serially, and stained as described previously. ${ }^{31}$

\section{Histoenzymatic studies}

Frozen sections were cut ( $10 \mu \mathrm{m}$ thickness) and placed on silanised slides. The sections were stained with a modified Gomori trichrome procedure. The sections were also stained for cytochrome $\mathrm{C}$ oxidase (COX), NADH dehydrogenase, and succinate dehydrogenase (SDH) (Bio-Optica Staining Kits; Bio-Optica, Milan, Italy). ${ }^{32}$

Electron microscopy

Tissue samples were fixed in Karnovsky solution and embedded in resin as previously reported. ${ }^{33}$ Ultrathin sections were stained with lead citrate and uranyl acetate, and examined with a Zeiss 902 electron microscope.

Biochemical assay

Activities of COX, NADH dehydrogenase, and $\mathrm{SDH}$ in whole heart homogenates were measured according to standard recommendations. ${ }^{34-36}$

\section{Controls}

Donor heart biopsy specimens and hearts excised at transplantation from patients with end stage ischaemic heart disease $(n=3)$, valvar heart disease $(n=4)$, and dilated 

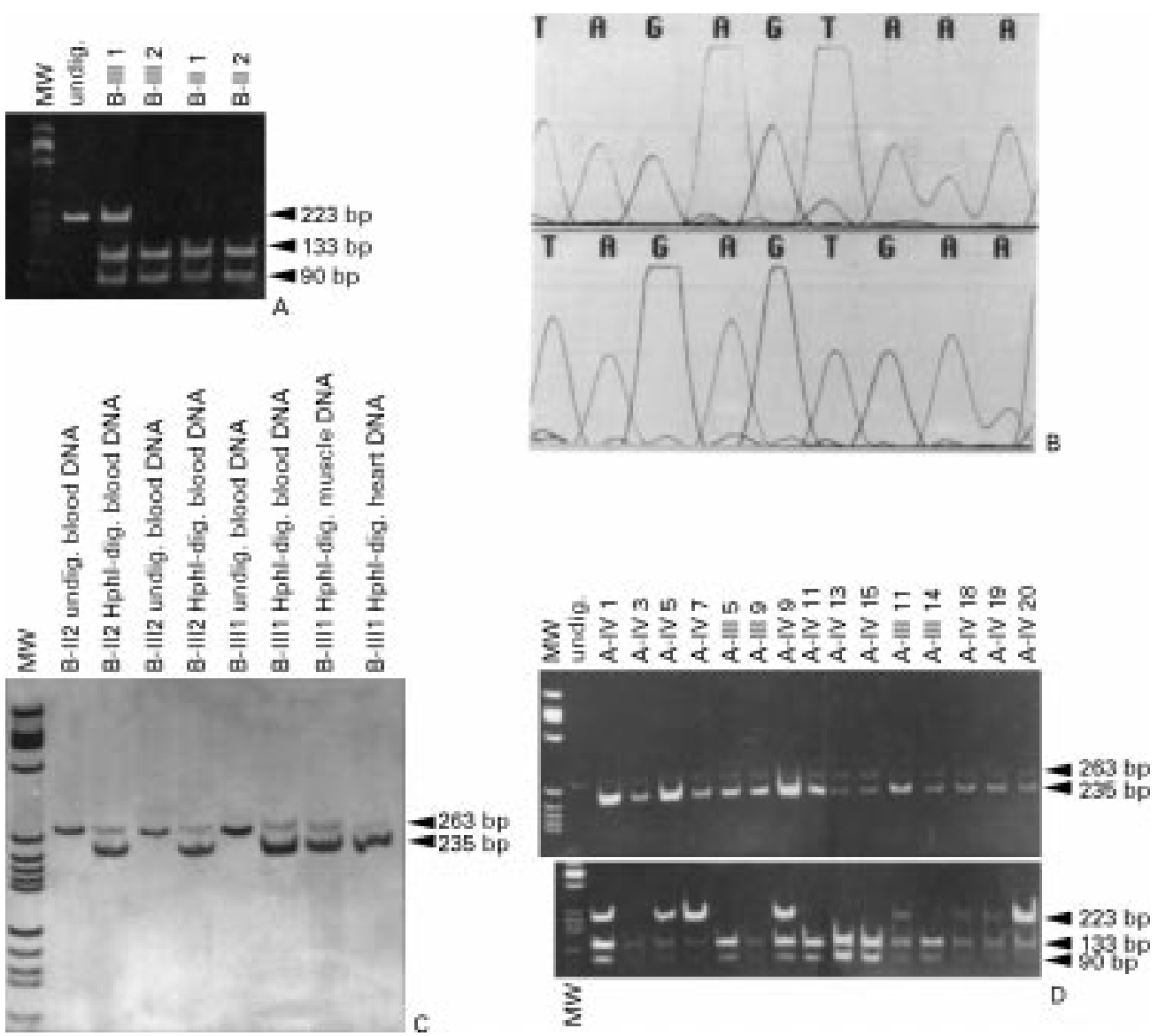

Figure 2 (A) Family B. PCR and restriction fragment length polymorphism (RFLP) analyses with EcoRI of the $\beta M H C$ gene region encompassing exon 9: molecular weight (MW) marker pBR 322 digested with HaeIII. EcoRI digestion of the mutant DNA produces a 223 base pair (bp) fragment, which corresponds to the mutated, uncut allele, and two fragments of 133 and $90 \mathrm{bp}$, which correspond to the mutated allele. (B) Family A. Top: wild-type DNA sequence 4294-4302 (TAGAGTAAA) of the 5' region of mtDNA tRNAIle from a normal control. Bottom: the A4300G (TAGAGTGAA) transition. (C) Family B. PCR and RFLP analyses using HphI to assess heteroplasmy of A4300G. MW marker pBR 322 digested with HaeIII. HphI digestion of the PCR fragment generated by the mismatch primer (A4297G) in combination with mutation A4300G produces two fragments of 235 and $28 \mathrm{bp}$ (the latter is not visible). The mutation is almost homoplasmic in heart mtDNA of proband III-1. (D) Family A-III and IV, live patients. Top: HphI digestion to assess heteroplasmy of the mtDNA A4300G transition. Severely affected patients IV-1, 5, and 9 have a very high amount of mutant DNA. Bottom: EcoRI digestion of the exon 9 region of $\beta M H C$. The mutation co-segregates with the phenotype, with the single exception of IV-7, for whom there was no echocardiographic evidence of hypertrophic cardiomyopathy and only negative $T$ waves in V1-V3 were seen on the ECG.

cardiomyopathies in which mtDNA sequencing excluded pathological mutation $(n=6)$ were used as controls for histoenzymatic and biochemical studies.

\section{Results}

BMHC GENE STUDY

G7981A transition was identified in probands A and B. A10428G transition was found in patients of kindred $\mathrm{C}$. These mutations were not identified in 24 unrelated patients with HCM studied in our laboratory (unpublished data), nor were they seen in the healthy controls.

A7981G transition (832 in cDNA) in exon 9 of probands A-IV-9 and B-III-1 created a nonconservative amino acid substitution of glutamine (Gln) for arginine (Arg) at position 249. The mutation produced a change of charge from +1 to $0(-1)$, and abolished a normally present EcoRI site (fig 2A and D). All echocardiographically affected patients of kindred A, whether symptomatic or asymptomatic, carried the $\beta M H C$ gene mutation.
Patient A-IV-7 with isolated T wave inversion in leads V1-V3 also had the same mutation.

The proband in kindred $\mathrm{B}$ had a de novo Arg249Gln mutation, which was not present in his maternal or paternal relatives. Paternity was assessed with a microsatellite STR ABI Prism STR primer set (Perkin Elmer), ${ }^{37}$ which includes the tetrameric STR loci: vWA31,TH01, F13A, and FES/FPS. The results showed that the proband inherited one allele from each parent for each of the four loci.

Both mother and son in kindred C carried an A to $G$ transition at position 10428 (1435 of cDNA) in exon 14. The mutation creates a non-conservative amino acid substitution of glutamic acid (Glu) for lysine (Lys) at position 450. The mutation resulted in a change of charge from +1 to $-1 \quad(-2)$. A forward mismatch primer (5'AGCCGCATCAATGCCACCCTGGAGCC3', mismatch underlined) (fig 3C) combined with the mutation generated a new AvaI site, which cleaved PCR fragments generated from the mutated 
A

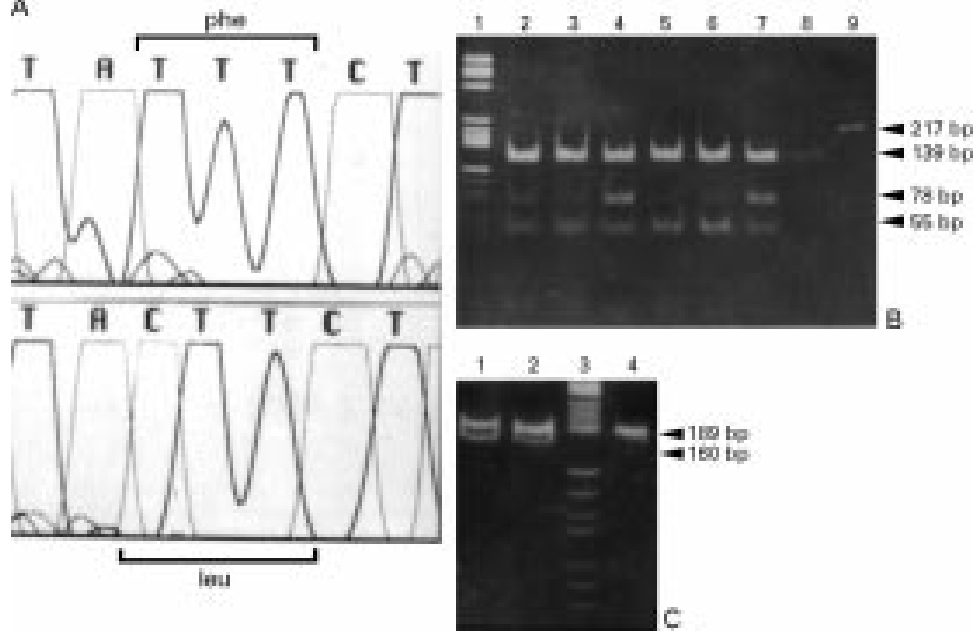

Figure 3 (A) Family C. Top: 9955-9961 wild-type sequence (TATTTCT) of the mtDNA COXIII gene from a normal control. Bottom: non-conservative T-C transition at position 9957 (TACTTCT) causes a Phe-Leu change. (B) PCR and restriction fragment length polymorphism (RFLP) analyses using RsaI digestion to detect heteroplasmy of mutation T9957C in the COX III gene; RsaI digestion of wild-type DNA produces two fragments of 139 and $78 \mathrm{bp}$, while RsaI digestion of the PCR product generated by the mismatch primer, in combination with the mutation, produces two further fragments of 55 and of $23 \mathrm{bp}$ (the latter is not shown). Lane 1: molecular weight marker PBR 322 digested with HaeIII; lanes 2, 3, and 4: patient II-2, heart, muscle, and blood specimens of patient II-2; lanes 5, 6, and 7: patient III-1 heart, muscle, and blood specimens of patient III-1, lane 8: normal control; lane 9: undigested control. (C) PCR and RFLP analyses with AvaI of the $\beta M H C$ gene region encompassing exon 14. Lane 3: $M W$ marker $p B R 322$ digested with HaeIII. AvaI digestion of the mutant DNA amplified with a forward mismatch primer (5' ACG CGC ATC AAT GCC ACC CTG GAG CC '3, mismatch underlined) produces a $189 \mathrm{bp}$ fragment, which corresponds to the wild-type, uncut allele, and two fragments of 160 and $29 \mathrm{bp}$ (the latter is not seen), which correspond to the mutated allele. Lanes 1 and 2: patients II-2 and III-1; lane 4: normal control. template into two fragments of 160 and 29 base pairs (bp).

mEDNA STUDY

Analogous to $\beta M H C$ gene mutation, mtDNA tRNA mutations in patients and maternal relatives in kindreds $\mathrm{A}$ and $\mathrm{B}$ were identical. mtDNA mutations in the hearts of patients in kindred $\mathrm{C}$ were identical and exclusive of those found in kindreds $\mathrm{A}$ and $\mathrm{B}$.

Automated DNA sequence analysis identified an $A$ to $G$ transition at position 4300 in the anticodon stem of the tRNAIle in kindreds $A$ and $\mathrm{B}$ (fig 2B). A forward mismatch primer (5'AGAGTTACTTTGATAGGG3', mismatch underlined) combined with the mutation generated a new HphI site; HphI cleaved the PCR fragments generated from the mutated template into two fragments of 235 and $28 \mathrm{bp}$ (fig $2 \mathrm{C}$ and $\mathrm{D}$ ). The mutation was heteroplasmic, with a nearly homoplasmic pattern in both proband hearts. The amount of mutant mtDNA was higher in the heart than in skeletal muscle and peripheral blood. It was also higher in affected patients than in asymptomatic relatives. At least $90 \%$ mutant DNA was required for manifestation of the disease. All maternal relatives of both A and B probands carried the heteroplasmic A4300G transition in tRNAIle; the percentage of heteroplasmy varied from $50 \%$ to $98 \%$. In addition, numerous mtDNA polymorphisms were identified, demonstrating

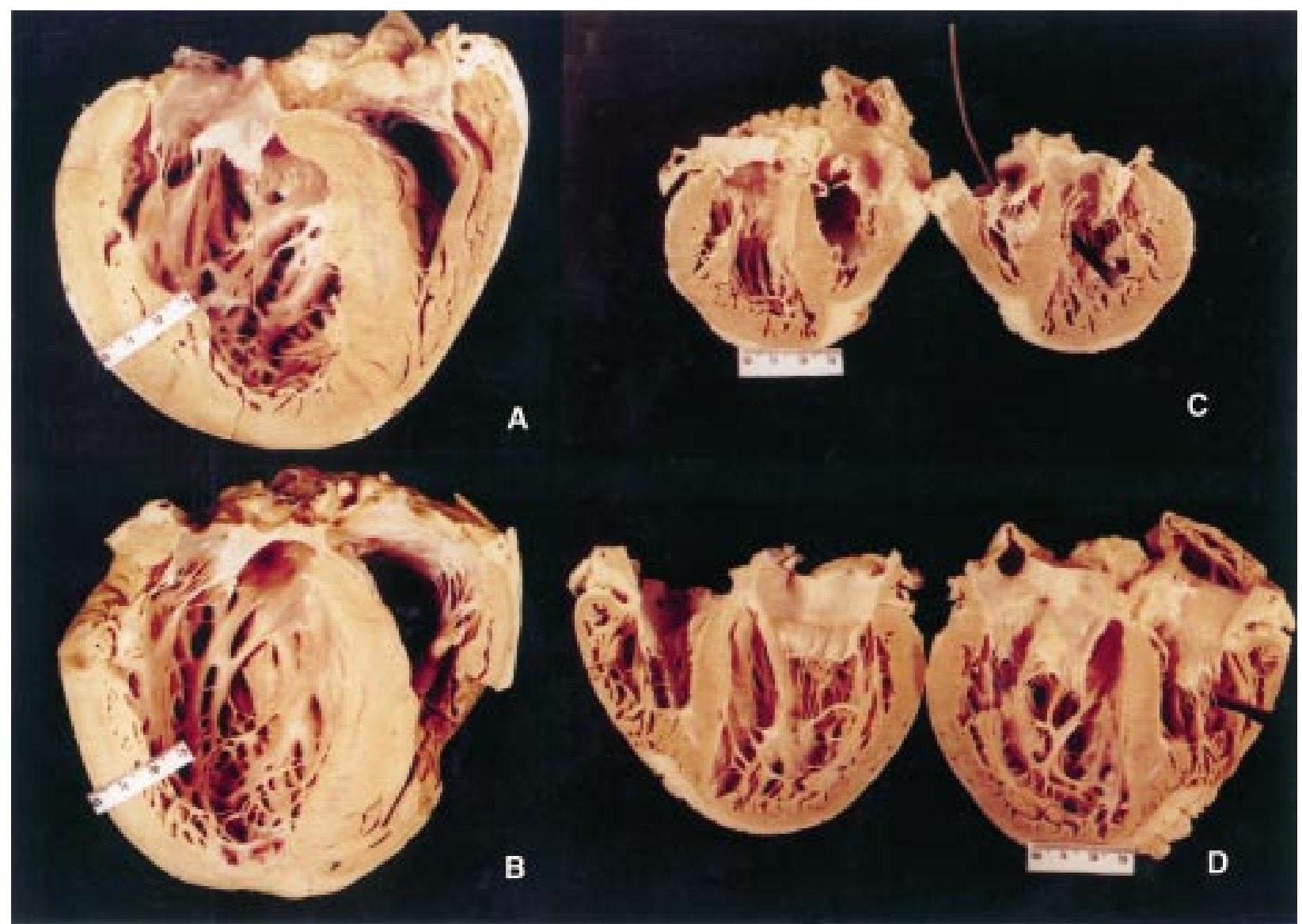

Figure 4 ( $A$ and B) Macroscopic views of the hearts excised at transplantation from the two probands of families $A$ and B. Note the similar macroscopic pattern, intraseptal scarring, ventricular thickness, and dilatation. (C and D) Macroscopic views of the hearts excised at transplantation from the affected mother and son of family C. Septal hypertrophy is asymmetrical and more prominent in the mother's heart (II-2, C) than in the son's heart (III-1, D). Table 1 lists the height and body weight of patients. 


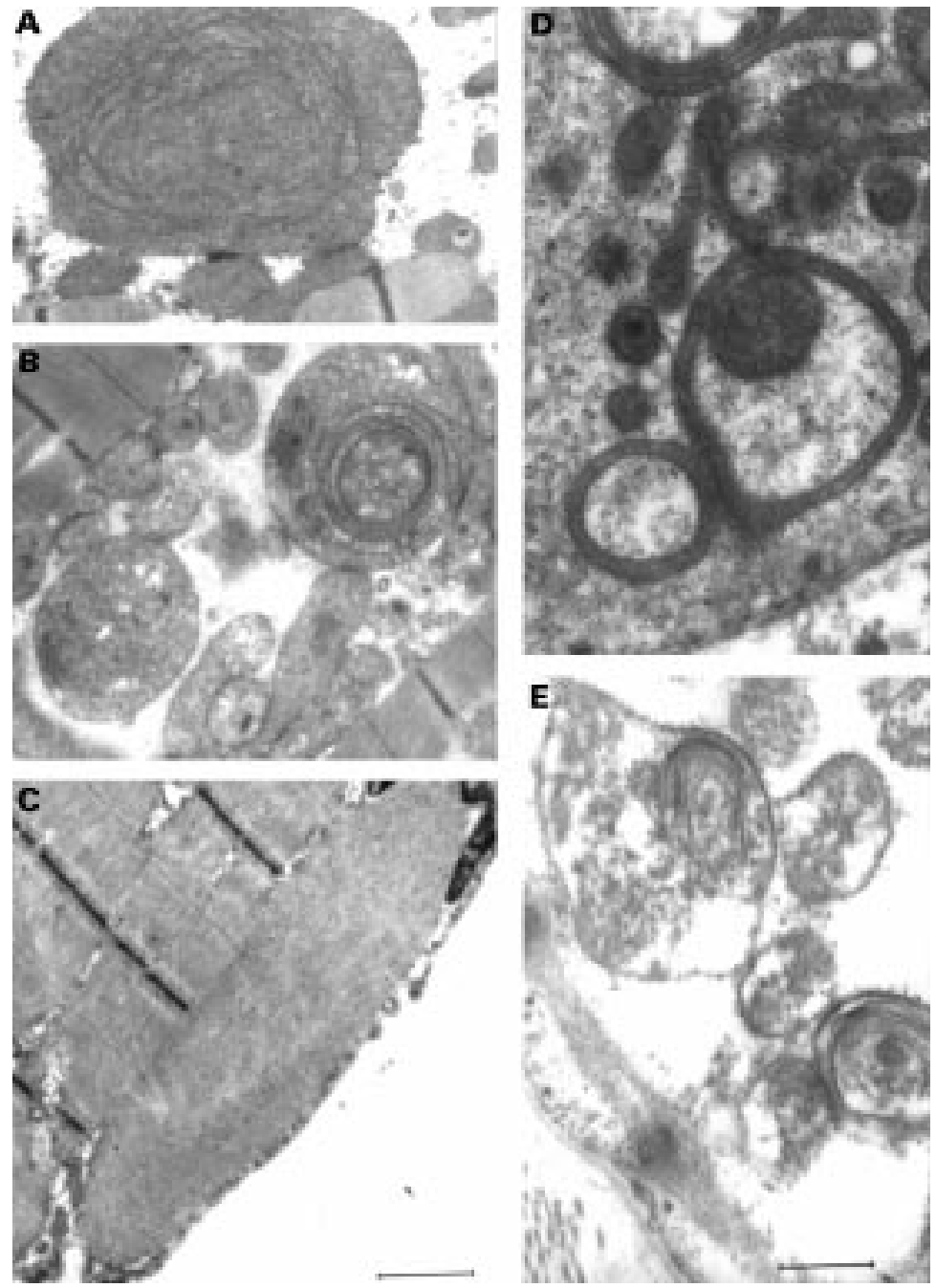

Figure 5 Electron micrographs of mitochondrial abnormalities. ( $A$ and B) Ring mitochondria, giant organelles with concentric cristae, and intramitochondrial inclusion bodies are seen in hearts of the two probands $A I V-9$ and B III-I (families $A$ and $B$ )

(C) Hyaline bodies were seen in all skeletal muscle samples from the three families. (E) Mitochondria in the heart of the mother (family C) affected by hypertrophic cardiomyopathy. Mitochondrial abnormalities in the heart of the mother and son of family $C$ were identical. (Stain: uranyl acetate, lead citrate.) (Bar $=1.1 \mu \mathrm{m}$ in $A-C$ and $0.6 \mu \mathrm{m}$ in $D$ and $E$.) minimal, homogenous, and fibrous. Ultrastructural studies showed an increased number of pleomorphic giant mitochondria with concentric cristae and osmiophilic inclusion bodies in the hearts of kindreds A and B. Mitochondria had concentric and tubular cristae with occasional osmiophilic inclusions in kindred C hearts (fig 5).

Histochemical characteristics of the mitochondrial respiratory chain enzymes in probands $A$ and $B$ with identical $m t D N A$ tRNA mutations were similar to each other but different from those of kindred $\mathrm{C}$, which carried a different mtDNA mutation in the CoxIII gene (fig 6). Patients with A4300G tRNAlle mutation had a severe COX deficiency $(\mathrm{COX}$ activity $=16$; mean reference value 155 (34). There was a negligible decrease in the activities of $\mathrm{COX}, \mathrm{NADH}$ dehydrogenase, and $\mathrm{SDH}$ in the hearts of kindred $\mathrm{C}$ with T9957C mutation in CoxIII gene.

Skeletal muscle samples stained with the modified Gomori trichromic method did not show ragged red fibres. SDH stain did not show pre-ragged fibres. COX, NADH dehydrogenase, and SDH stain patterns were normal as was mitochondrial enzyme activity. Some muscle fibres had rare centralised nuclei and sparse non-specific lysis and damage of the myofilaments; ultrastructural examination showed occasional subsarcolemmal hyaline bodies (fig 5C). Mitochondria did not increase in number and showed focal non-specific patterns of cristolysis.

\section{Discussion}

The present study demonstrates the coexistence of $\beta M H C$ gene missense mutations and heteroplasmic mtDNA mutations in patients with HCM and late CHF. Patients sharing identical mutations had similar clinical histories, morphological features, and histochemical characteristics. The amount of mutated mtDNA segregated with the CHF phenotype and was higher in the myocardium than in skeletal muscle or peripheral blood. It was also higher in affected patients than in asymptomatic relatives.

Both $\beta M H C$ gene and mtDNA mutations seen in families A and B have been causally linked to HCM. ${ }^{8-19}$ Mutation Arg249Gln was detected in a Canadian family, ${ }^{8}$ while A4300G mtDNA tRNAIle was described in an Italian family. ${ }^{19} \mathrm{mtDNA}$ in the Canadian family and the $\beta M H C$ gene in the Italian family were not analysed. Prerequisites for pathogenicity are present in the mutation Arg249Gln: the mutant residue has been strongly conserved throughout evolution and it has not varied in any vertebrate muscle myosins (Swissprot Database). ${ }^{38}$ In the Canadian family, the mutation co-segregated with the disease and showed an age related penetrance: only one of nine affected family members with the mutation was younger than 20 years. $^{8}$ In our series, family members with mutation Arg249Gln had an age related penetrance. Accordingly, seven of eight patients older than 20 years had HCM; the remaining one had electrocardiographic negative $\mathrm{T}$ waves in leads V1-V3. In contrast, family members 
younger than 20 years had no evidence of the disease. Furthermore, the outcome of affected family members in kindred A was similar to that of the Canadian kindred; 10 of 21 patients $(48 \%)$ of the Canadian family died of HCM, with four sudden deaths. Similarly, of 11 affected members of kindred $\mathrm{A}$, two died suddenly, two died of $\mathrm{CHF}$, one underwent cardiac transplantation, and one had life threatening ventricular tachyarrhythmias. Two possibly affected patients (A-III-15 and III-17) died of unspecified cardiac failure in their 50s.

One family member was affected in kindred B. Maternal and paternal relatives had normal electrocardiographic and echocardiographic findings. The mutation appeared to be de novo and neither parent had mutation Arg249Gln. Maternity was assessed by mtDNA study and paternity with a specific microsatellite panel. Therefore we hypothesise that G7981 is a hot spot region. De novo $\beta M H C$ gene mutations in sporadic HCM have been reported by Watkins et $a l .{ }^{2}$ The finding of an identical mutation in sporadic HCM (our B patient) as well as in familial HCM (our A and QQ Canadian kindreds) supports further the hypothesis that the mutation itself has a role in the pathogenesis of the disease.

Detection of the A4300G transition in mtDNA tRNAIle in families A and B suggests that this mutation also plays a part in the pathogenesis of the disease: adenine at position 4300 has been highly conserved throughout evolution $^{19}$ and the mutation is heteroplasmic. A higher proportion of mutated DNA was found in affected patients with an almost homoplasmic pattern in the affected end organ. COX activity and stain intensity were also considerably lower than in the controls. Normal staining was seen only with occasional myocytes, analogous to that observed in ragged red fibres in skeletal muscle from patients with mitochondrial encephalomyopathy lactic acidosis and stroke-like syndrome (MELAS). ${ }^{39}$ Myocytes from patients with mutation A4300G had increased numbers of mitochondria, as well as an increase in their size and altered morphology (concentric and abnormally arranged cristae). It seems likely that the A4300G mtDNA mutation, which lies in the anticodon stem of tRNAIle, does not severely affect isoleucine transfer, so a very high amount of mutated DNA is necessary to cause deficient expression of COX. Finally, this mutation has been reported as a causative mutation responsible for cardiomyopathy in an Italian patient with an identical phenotype. ${ }^{19}$

The described findings support the hypothesis that mutation Arg249Gln in the $\beta M H C$ gene could be the true determinant of the hypertrophic phenotype, while mtA4300G could affect energy supply, resulting in CHF. This hypothesis is most likely in hypertrophic hearts in which the mutant protein negatively influences activity of the wild-type one (dominant negative pathogenic hypothesis). ${ }^{11}$ The amount of mutated mtDNA, its progressive accumulation with aging, and age dependent penetrance of Arg249Glu could explain why young patients with both mutations may not present the phenotype.
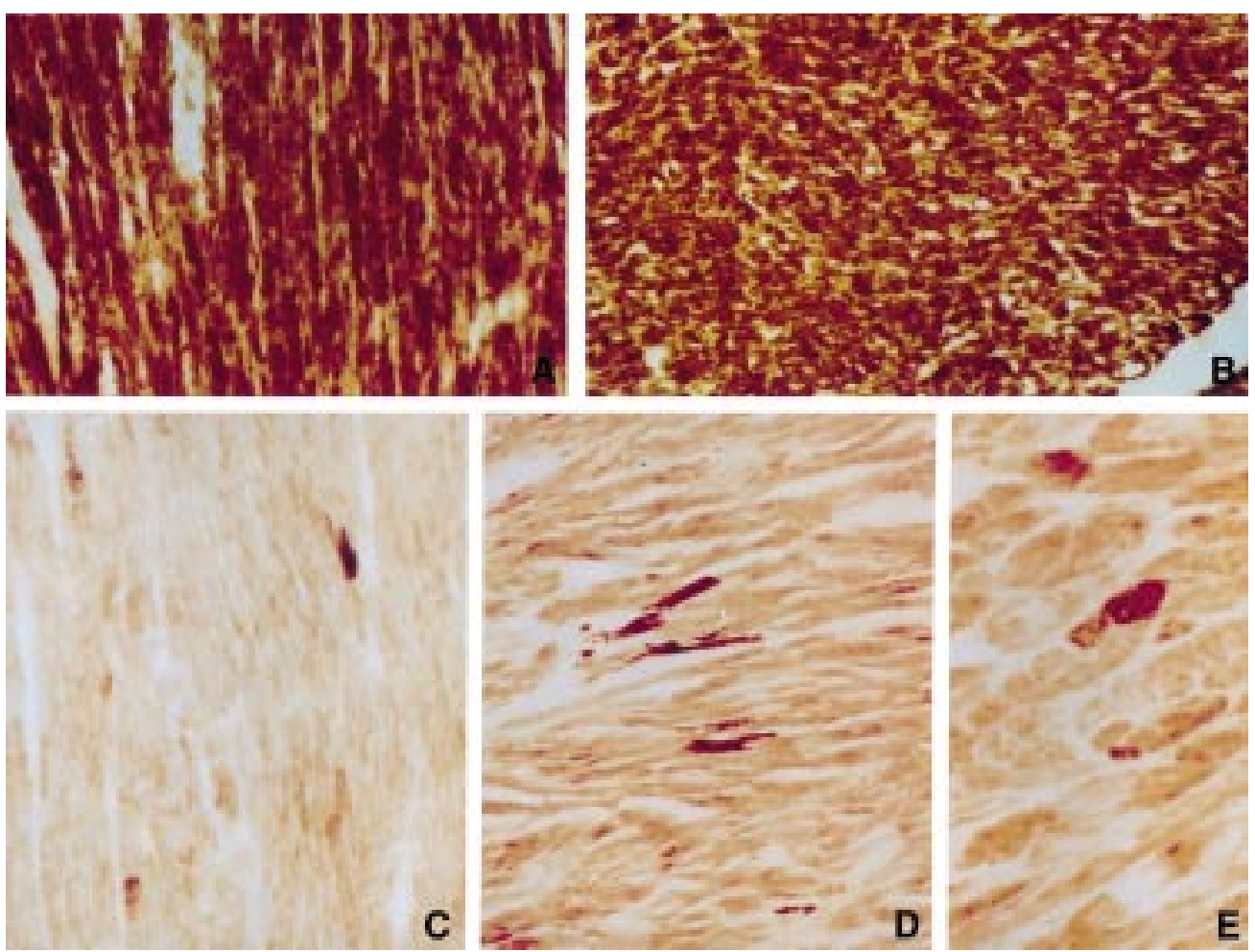

Figure 6 Light micrographs showing normal histochemical reactions for COX. (A) Donor heart biopsy specimen (original magnification $\times 160$ ). (B) Heart of a patient with non-mitochondrial cardiomyopathy excised at transplantation (magnification $\times 120)$. (C-E) Histochemical reaction for COX in B-III-1, A-VI-1, and A-VI-9 compared with that in controls. The samples show a severe decrease in intensity of COX staining in most myocytes: very few fibres retain normal $C O X$ reaction (magnification $C$ and $D \times 120$, and $E \times 180$ ). 
A major limitation of the study lies in the fact that the percentage of mutated mtDNA could not be studied in the hearts of mutated relatives with the subclinical phenotype as an invasive procedure such as endomyocardial biopsy may be unethical in asymptomatic patients. However, the mtDNA mutation alone was not associated with disease nor the $\beta M H C$ mutation alone. Family members exclusively carrying the $\beta M H C$ mutation are healthy but young (less than 20 years). Accordingly, the question of whether mtDNA mutation is important for disease will probably be clarified by follow up. It is pertinent to discuss patient A-IV-7 who is 39 years of age and has both $\beta M H C$ and mtDNA mutations. Her echocardiogram is within normal limits but she has negative $\mathrm{T}$ waves in leads V1-V3. A low percentage of mutated mtDNA in her heart could partially explain the absence of disease. She has three healthy sons: one has both $\beta M H C$ and $\mathrm{mtDNA}$ mutations and the remaining two have only mtDNA mutations. The child with both mutations is younger than 20 years. The absence of disease in all A4300G maternal relatives of our sporadic proband $\mathrm{B}$ makes it unlikely that this mutation is, by itself, pathogenic and the abundance of the mutation in affected hearts (nearly homoplasmic) makes it a co-determinant in the congestive evolution of the disease.

Identical considerations apply to the $\beta \mathrm{MHC}$ Lys450Glu mutation detected in family $\mathrm{C}$. The mutant lysine residue has been highly conserved throughout evolution, is invariant in all vertebrate muscle myosins (Swissprot Database), ${ }^{38}$ and is not present in controls. The rather malignant effect of this mutation may be explained by the change of charge from +1 to $-1(-2) .{ }^{411}$ The role of the mtDNA T9957C (CoxIII gene) missense mutation is less clear. This mutation is associated with a MELAS phenotype. ${ }^{40}$ Prerequisites for pathogenicity are present: a highly conserved position, heteroplasmia, and absence from controls. The mutation was associated with a mitochondrial proliferation of microvasculature cells in muscle ${ }^{40}$; however, this finding was absent in our patients. The proportion of mutated DNA may have a major role in our phenotypes, as found in the skeletal muscle of the patient with MELAS. ${ }^{40}$ However, given that our informative IV- 1 patient is affected, the $\beta$ MHC Lys $450 \mathrm{Glu}$ mutation seems to result in a hypertrophic phenotype; obviously, she did not inherit the paternal mtDNA mutation. The child is very young and has no signs of $\mathrm{CHF}$. The affected III-3 female, like her brother, is likely to carry both $\beta M H C$ gene and mtDNA mutations. These observations raise intriguing questions, and follow up of the child will help to exclude or confirm mtDNA mutation as a potential cofactor in the late congestive evolution of HCM.

CONCLUSIONS

The genetic heterogeneity of HCM seems to be more complex than currently suspected and the spectrum of penetrance or malignancy of $\beta M H C$ mutations is still unclear. Nevertheless, the important message from our study is that more than one gene or factor may contribute to the pathogenesis of HCM. This finding could help to explain why, even within a single family, the clinical behaviour of HCM is so heterogeneous, and why the age related penetrance of $\beta M H C$ mutations alone does not fully explain the phenotypic characteristics. The high degree of heteroplasmy of mtDNA mutations found in the heart may contribute to the pathogenesis of CHF complicating HCM.

The authors are indebted to Drs P Fortina and S Surrey, the Children's Hospital of Philadelphia, University of Pennsylvania School of Medicine, Philadelphia, and to M Devoto, Rockefeller University, New York, for their helpful revision of the manuscript. This study was supported by cardiovascular pathology script. This study was supported by cardiovascular pathology Pavia, Italy.

1 Maron BJ, Nichols PF, Pickle LW, et al. Patterns of inheritance in hypertrophic cardiomyopathy: assessment by ance in hypertrophic cardiomyopathy: assessment by Cardiol 1984;53:1087-94.

2 Watkins H, Thierfelder L, Hwang D, et al. Sporadic hypertrophic cardiomyopathy due to de novo myosin mutations. Clin Invest 1992;90:1666-71

3 Marian AJ, Yu Q, Mares A Jr, et al. Detection of a new mutation in the $\beta$-myosin heavy chain gene in an individual with hyperthophic cardiomyopathy. $\mathcal{F}$ Clin Invest 1992;90: 2156-65.

4 Watkins $\mathrm{H}$, Rosenzweig A, Hwang D, et al. Characteristics and prognostic implications of myosin missense mutations in familial hypertrophic cardiomyopathy. $N$ Engl $7 \mathrm{Med}$ 1992;326:1108-14.

5 Fananapazir L, Dalakas MC, Cyran F, et al. Missense mutations in the $\beta$-myosin heavy-chain gene cause central core disease in hypertrophic cardiomyopathy. Proc Natl Acad Sci USA 1993;90:3993-7.

6 Watkins H, Thierfelder L, Anan R, et al. Independent origin of identical $\beta$ cardiac myosin heavy-chain mutations in
hypertrophic cardiomyopathy. Am f Hum Genet 1993;53: hypertrop

7 Moolman J, Brink PA, Corfield VA. Identification of a new missense mutation at Arg403, a CpG mutation hotspot, in exon 13 of the $\beta$-myosin heavy chain gene in hypertrophic cardiomyopathy. Hum Mol Genet 1993;2:1731-2.

8 Rosenzweig A, Watkins H, Hwang DS, et al. Preclinical diagnosis of familial hypertrophic cardiomyopathy by genetic analysis of blood lymphocytes. N Engl f Med 1991; 325:1753-60.

9 Anan R, Greve G, Thierfelder L, et al. Prognostic implications of novel $\beta$ cardiac myosin heavy chian gene mutations that cause familial hypertrophic cardiomyopathy. $\mathcal{F}$ Clin Invest 1994;93:280-5.

10 Rayment I, Holden HM, Sellers JR, et al. Structural interpretation of the mutations in the $\beta$-cardiac myosin that have been implicated in familial hypertrophic cardiomyopathy. Proc Natl Acad Sci USA 1995;92:3864-8.

11 Vikstrom KL, Leinward LA. Contractile protein mutations aikstrom KL, Leinward LA. Contractile protein muta

12 Bonne G, Carrier L, Bercovici J, et al. Cardiac myosin binding protein-C gene splice acceptor site mutation is associated with familial hypertrophic cardiomyopathy. Nat Genet 1995;11:438-40.

13 Thierfelder L, Watkins H, MacRae C, et al. $\alpha$-tropomyosin and cardiac troponin $\mathrm{T}$ mutations cause familial hypertrophic cardiomyopathy: a disease of the sarcomere. Cell 1994;77:701-12

14 Coviello DA, Maron BJ, Spirito P, et al. Clinical features of hypertrophic cardiomyopathy caused by mutation of a "hot spot" in the alpha-tropomyosin gene. $7 \mathrm{Am}$ Coll Cardiol 1997;29:635-40.

15 Watkins H, McKenna WJ, Thierfelder L, et al. Mutations in the genes for cardiac troponin $\mathrm{T}$ and $\alpha$-tropomyosin in hypertrophic cardiomyopathy. $N$ Engl $\mathscr{f}$ Med 1995;332: 1058-64.

16 Poetter K, Jiang H, Hassanzadeh S, et al. Mutation in either the essential or regulatory light chains of myosin are associthe essential or regulatory light chains of myosin are associated with a rare myopathy in h
muscle. Nat Genet 1996;13:63-9.

17 Zeviani M, Gellera G, Antozzi C, et al. Maternally inherited myopathy and cardiomyopathy: association with mutation in mitochondrial DNA tRNA(Leu)(UUR). Lancet 1991; 338:143-7.

8 Merante F, Tein I, Benson L, et al. Maternally inherited hypertrophic cardiomyopathy due to a novel T-to-C transition at nucleotide 9997 in the mitochondrial tRNAglycine gene. Am f Hum Genet 1994;55:437-46.

19 Casali C, Santorelli F, D'Amati G, et al. Novel mtDNA point mutation in maternally inherited cardiomyopathy. Biochem Biophys Res Commun 1995;213:588-93.

20 Merante F, Myint T, Tein I, et al. An additional mitochondrial tRNAIle point mutation (A-to-G at nucleotide 4295) causing hypertrophic cardiomyopathy. Hum Mutat 1996;8:216-22.

21 Taniike M, Fukushima H, Yanagihara I, et al. Mitochondrial tRNAIle mutation in fatal cardiomyopathy. Biochem Biophys Res Commun 1992;186:47-53. 
22 Santorelli FM, Mak S, El-Schahawi M, et al. Maternally inherited cardiomyopathy and hearing loss associated with (G8363A). Am f Hum Genet 1996;58:933-9.

23 Santorelli FM, Mak S, Vazquez-Aceredo M, et al. A novel mitochondrial DNA point mutation associated with mitochondrial encephalomyopathy. Biochem Biophys Res Commun 1995;216:835-40.

24 Obayashi T, Hattori K, Sugiyama S, et al. Point mutations in mitochondrial DNA in patients with hypertrophic cardiomyopathy. Am Heart f 1992;124:1263-9.

25 Silvestri G, Santorelli FM, Shanske S, et al. A new mtDNA mutations in the tRNALeu(UUR) gene associated with maternally inherited cardiomyopathy. Hum Mutat 1994;3: 37-43.

26 Sambrook J, Fritsch SF, Maniatis T. Molecular cloning: a laboratory manual. Cold Spring Harbor, New York: Cold Spring Harbor Laboratory, 1989:9.14-9.21.

27 Myers RM, Maniatis T, Lerman LS. Detection and localization of single base change by denaturing gradient gel election of single base change by denaturing gradient
trophoresis. Methods Enzymol 1987;155:501-27.

28 Sartore M, Grasso M, Piccolo G, et al. Leber's hereditary optic neuropathy (LHON)-related mitochondrial DNA sequence changes in Italian patients presenting with sporadic bilateral optic neuritis. Biochem Mol Med 1995;56 45-51.

29 Jaenicke T, Diederich KW, Haas W, et al. The complete sequence of the human beta-myosin heavy chain gene and a comparative analysis of its products. Genomics 1990;8: 194-206.

30 Anderson S, Bankier AT, Barrel BG, et al. Sequence and organization of the human mitochondrial genome. Nature 1981;290:457-65.
31 Arbustini E, Pucci A, Grasso M, et al. Expression of natriuretic peptide in ventricular myocardium of failing human hearts and its correlation with the severity of clinical and heamodynamic impairement. Am f Cardiol 1990;66:97380

32 Block JT, Judge D, Demers L, et al. Ragged red fibers. A biochemical and morphological study. $\mathcal{F}$ Neurol Sci 1975;26:479-88.

33 Patella V, Marino I, Lamparter B, et al. Human heart mast cells. Isolation, purification, ulrastructure and immunological characterization. F Immunol 1995;154:2855-65.

34 Sottocasa GL, Kuylenstierna B, Ernster L, et al. An electron transfer system associated with the outer membrane of the mitochondria. f Cell Biol 1967;32:415-38.

35 King TE, Howard RL. Preparation and properties of soluble $\mathrm{NADH}$ dehydrogenase from cardiac muscle. Methods Enzymol 1967;10:322-31.

36 King TE, Howard RL. Preparation of succinate dehydrogenase and reconstitution of succinate oxidase. Methods Enzymol 1967;10:322-31.

37 Edwards A, Hammond HA, Jin L, et al. Genetic variation at the five trimeric and tetrameric tandem repeat loci in four human population groups. Genomics 1992;12:241-53.

38 Bairoch A, Apweiler R. The SWISS-PROT protein sequence data bank and its new supplement TrEMBL. Nucleic Acids Res 1996;24:21-5.

39 Koga Y, Nonaka I, Sunohara N, et al. Variability in the activity of respiratory chain enzymes in mitochondrial myopathies. Acta Neuropathol (Berl) 1988;76:135-41.

40 Manfredi G, Schon EA, Moraes CT, et al. A new mutation associated with MELAS is located in a mitochondrial DNA polypeptide-coding gene. Neuromuscul Disord 1995;5:391-8. 